\title{
INTERACTIVE SUPPORT SYSTEM USING HUMANOID ROBOT FOR REHABILITATION OF GROSS MOTRICITY IN CHILDREN
}

\author{
Piedad A. Semblantes, Pilatasig Marco \\ Universidad de las Fuerzas Armadas ESPE, Sangolquí, Ecuador \\ \{pasemblantes, mapilatagsig\} @espe.edu.ec
}

\begin{abstract}
The present work is based on the implementation of a human-robot HRI interaction system to support the sessions of gross motor rehabilitation in infants. The proposal is formed by a humanoid robot, which indicates the exercise of recovery that the child must imitate through movements of their extremities. At the same time, the mechanism visually and auditorily motivates the child to ensure that the proposed exercises are carried out in a correct manner and thus assess the child's progress in therapy. A FieldProgrammable Gate Array (FPGA) is used for the architecture of the system, which is responsible for controlling the robot as well as the validation algorithm and an auxiliary processor for the acquisition and processing of the information taken from the sensors in real time. For the validation of the exercises proposed in the therapy, the system proposes an algorithm based on classifiers. The algorithm is based on the comparison of patterns of the exercises performed by the humanoid in contrast to the acquired movements of inertial measurement sensors subject to the user.
\end{abstract}

Keywords: Humanoid Robot, FPGA, DTW, Rehabilitation Systems, Gross Motricity, Children.

Palabras clave: Robot humanoide, Matriz de puertas programables FPGA,

Alineamiento temporal dinámico DTW, Sistemas de rehabilitación, Motricidad Gruesa, Niños.

\section{Introduction}

According to the World Health Organization, (WHO) more than 1 billion people, or $15 \%$ of the world's population, have some kind of disability. In a more isolated case and depending on the type of limitation, children with disabilities usually have difficulties in carrying out daily activities, low educational performance, fewer economic opportunities, and a higher rate of poverty in adulthood compared to healthy individuals. [1]. At the local level and according to records from the National Institute of Statistics and Census (INEC) of Ecuador, there is a growing demand in health centers in the area of physiotherapy, reporting in recent years a rate of four million treatments performed 
along the region [2][3]. The need for therapy in people who have suffered an injury or illness causes a substantial increase in patients going to health centers, which requires a considerable investment of time and money. Another kind of problem is the rehabilitation of children, due to the emotional state of their age. In these cases, traditional rehabilitation sessions are often presented as unsuccessful and tedious therapies, because these processes tend to be routine and boring, causing children not accostum to them and therefore slow down their recovery [4]. For this reason, the use of new methods based on emerging, innovative, and motivating technologies in the traditional rehabilitation of children, promotes better results in their recovery. In this way, new methods to treat motor problems caused by cerebral palsy, brain injury or stroke have been effective.Virtual interfaces have been developed, which can be manipulated by means of innovative technological instruments such as gloves or robotic arms, thus forming systems capable of providing support in the recovery of motor disabilities and attracting the attention and curiosity of children [5] [6] [7].

On the other hand, robotics has been solving problems in different areas, both industrial and consumer. In this context, robotic prototypes aimed to physical assistance in an invasive way (prosthesis or exoskeletons) or social assistance in a non-invasive way (robots for guidance, supervision, and diagnostic of therapy) merge engineering with health areas [8]. In this context, the non-invasive robotic prototypes do not intend to apply forces on the extremities of children, but rather emphasize attracting the attention of the child, either by guiding or replicating the movements of the robot as he/she would be with other children. In this case, [9], [10], and [11] propose humanoid robotic mechanisms to support the treatment of children with autism. In the same way, works dedicated to treating motor problems have been developed. For example, [12] presents a robot developed to rehabilitate the upper limbs by sequelae left by stroke (CVA). The work presents a technique of non-invasive therapy, in which the robot does not come into contact with the patient. The autonomy of the robot provides monitoring and recording of activities, besides encouraging the patient of the exercise routine of their recovery program. In conclusion, the programs of motor rehabilitation in children have proven, through exhaustive studies, to be very useful in the recovery of their functions and the improvement of their quality of life [13].

Therefore, this work proposes a novel alternative which provides support to the tedious rehabilitation sessions for minors, in order to present a game environment where the patient interacts physically with a humanoid robot, which motivates and guides the child in the execution of the therapy. Through a series of movements which the robot executes as guide exercises, it is intended to rehabilitate and strengthen the locomotor system coordinated by the cerebral cortex and secondary structures that modulate it, the same ones that have been affected by injuries left, either by a brain accident, illness or damage by physical activities. To achieve this, the proposed system carries out a validation algorithm for the exercises performed by the child, taking into account the techniques of pattern identification given by the DTW algorithm (dynamic temporal alignment). The purpose of this algorithm is to measure the homogeneity of the sequences obtained from a set of electronic armbands arranged in such a way in the injured extremities of the minor (3 SPACE SENSOR) versus the pre-recorded pattern sequence 
in the humanoid robot (BIOLOID ROBOT). Finally, several experimental results are shown to validate the proposed system.

\section{System Structure}

The developed system is based on a human-robot interaction (HRI) architecture, where the patient interacts directly with a humanoid robot. The rehabilitation proposal is based on the comparison between the therapeutic movements made by the robot versus the movements replicated by the patient. These activities are detected through the use of inertial measure sensors, which are located on the affected limbs which are to be rehabilitated in the child. On the other hand, the guiding movements which the robot executes are achieved through control criteria based on inverse kinematics. The Fig. 1 shows the scheme of the developed system. The proposal includes a rehabilitation strategy that can be used both in upper and lower extremities, where the progress of the therapy will be assessed by degrees of difficulty (moderate and mild) based on the initial medical diagnosis. Additionally, the system provides feedback through audio outputs which motivate and guide the child during the execution of the treatment.

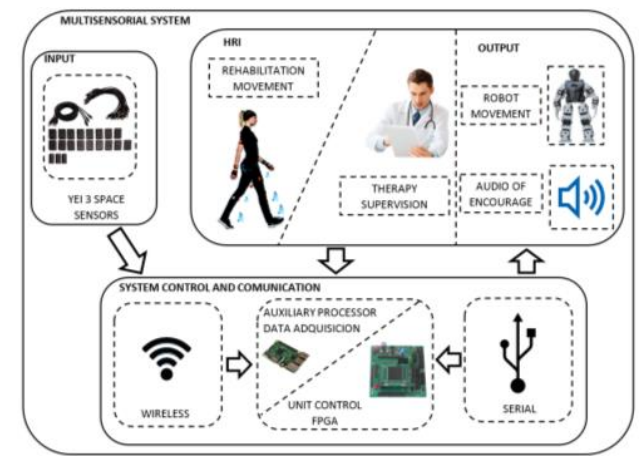

Fig. 1. Structure of the System for Thick Motricity Rehabilitation

The interaction of the patient with the rehabilitation robotic system is based on the information acquired from the inertial measure sensors (IMU). On the other hand, the movements of the robot are in function to the previous diagnosis of the therapist, who evaluates the child and designates the exercises that must be carried out in the therapy. This operating scheme splits the system into five parts: system inputs, auxiliary processor architecture, control algorithm design, outputs, and user interface, as shown in Fig. 2.

The a) inputs of the system, they are electronic devices in charge of capturing signals from a physical medium, to later be interpreted and execute actions. This research uses a set of 10 sensors 3-SPACE-SENSOR WIRELESS, formed by gyroscopes, accelerometers and triaxial compasses that give the system information about speed, acceleration and gravitational forces of the ex-extremes tested. The most relevant characteristics of this type of sensors are their autonomy and high performance since they use $2.4 \mathrm{GHz}$ 
wireless communication, providing the system with low latency and high precision at the time of data capture in real time.

b) architecture of the auxiliary processor, it has been considered to use a low cost card as a device responsible for carrying out the following processes: i) acquisition of data, in this stage the information of an antenna which interconnects all the inertial sensors through wireless communication with spread-spectrum protocol (DSSS), obtaining temperature information, accelerometers, gyroscopes, and compasses (Fig. 3). ii) Data processing, in this stage, through the programming of a library in Python the information provided by the antenna is read and unpacked for later processing and through direct kinematics obtain the position of the extremities with respect to a fixed point in real time. iii) Voice feedback, this part uses free software based on Linux which can convert plane text into audio outputs, with the purpose of reproducing guide phrases and motivation during the execution of the therapy. iv) Data output, finally in this stage the information of the sequences is framed as a function of position vectors in $x, y, z$, to be sent to the control unit commanded by an FPGA, by means of serial communication.

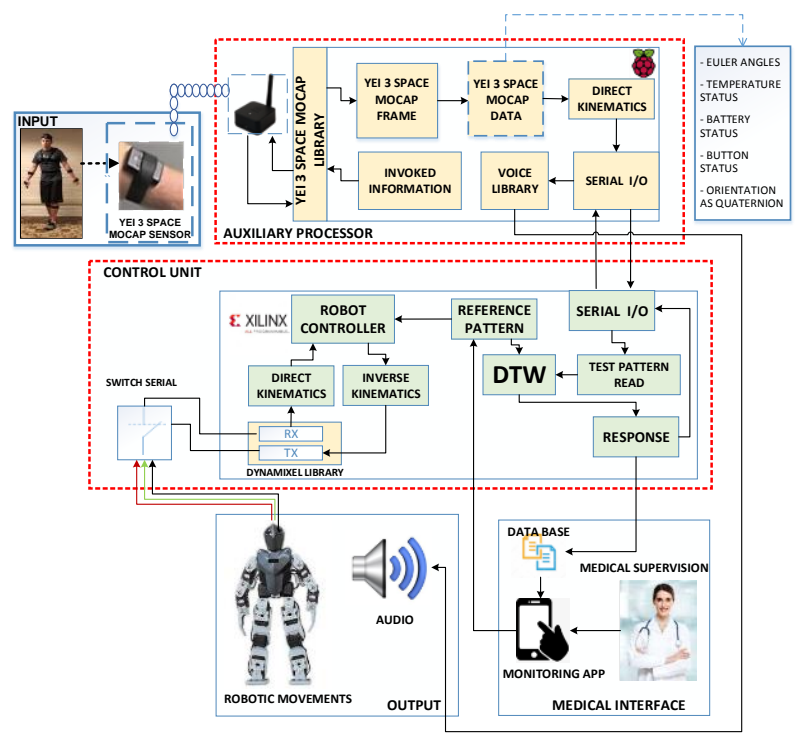

Fig. 2. Operative Scheme

The c) control unit, is presided over by an FPGA, where the control architecture is developed both for the positioning of the extremities of the humanoid robot and for the algorithm which validates the patient's movements. Based on the above and considering that there are vectors of reference movements pre-recorded in the FPGA, the following stages are detailed: i) execution of the controllers for the extremities of the humanoid, where the motion reference vectors are used as desired inputs to the controllers. As detailed in Section III, the controllers tend to reach the desired point through the use of basic techniques, where each of the extremities (each described with three degrees of 
freedom) must generate repeatable movements for the child. The FPGA includes internal libraries that command the chain of intelligent servomotors, as well as read positioning information to validate the execution and provide errors that will be corrected by the control strategy. On the other hand, the reference vectors are used by the DTW algorithm (an algorithm for each extremity) with the objective of ii) compare them with the vectors generated by the patient, after a session is finished or when the application has reached a timeout. Finally, iii) the FPGA can reply to serial communication commands, with the purpose of providing responses of the comparison of patterns, validating or not the successful execution of the session. Likewise, these responses are sent forcibly to the auxiliary preprocessor in order to generate sonorous commands that encourage the patient.

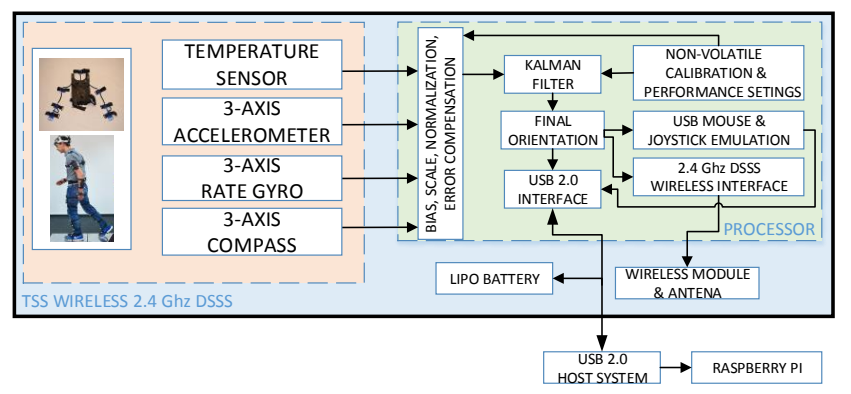

Fig. 3. Acquisition of data with 3-SPACE sensors

The elements used as $d$ ) outputs for this system are described below: i) the humanoid robot reproduces a series of movements by positioning its dynamixel motors, these movements are generated by the FPGA and are in function of the initial requirements of the therapist when evaluating the patient. ii) Audio, it is used to guide and to encourage the patient while it develops the exercises of rehabilitation, these phrases are generated by the auxiliary processor and reproduced by a speaker located on the robot humanoid.

The e) user interface, aims at the interaction of the therapist with the rehabilitation system through a menu of options which allow the user to select the routine to be performed, to save the results of the execution of the patient's exercises, as well as enable the storage of new rehabilitation movements, in order to provide flexibility to the proposed system (Fig. 4). 


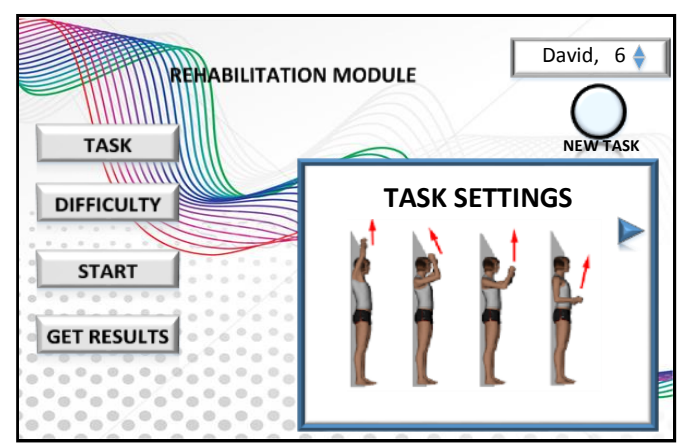

Fig. 4. User's interface

\section{Proposal development}

\subsection{Reading of patient movements}

The location of the patient's points of interest (hands and feet) is stablished through the use of inertial sensors as shown in previous chapters. The placement of the sensors is shown in Fig. 5. By means of these sensors, it is possible to identify the angle in which the segment of the limb is located and by means of the geometric model the position of the points of interest is located. For this case, the sensors located in the upper extremities are placed on the biceps and on the forearm; while in the lower extremities they are located on the quadricep and the tibialis anterior. For each sensor, two Euler angles are obtained, the one which rotates on the $X$ axis and the other one that rotates on the $Z$ axis.

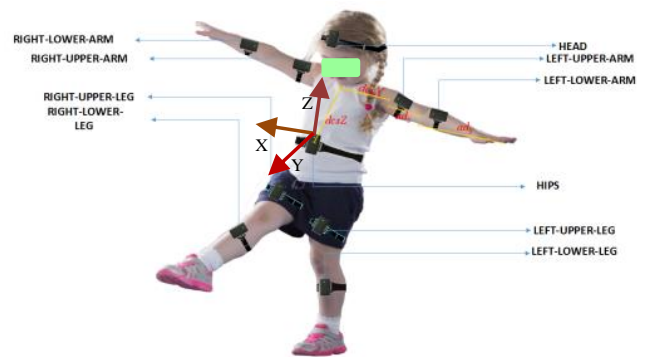

Fig. 5. Distribution of the 3 Space Sensors

Remark 1: The lower extremity (forearm or anterior tibial muscle) can rotate independently on the $Z$ axis, therefore, it is considered as an additional parameter to obtain the final displacement of the point of interest.

The direct kinematic model allows to obtain the current position of the points of interest given the entry angles. Considering four degrees of freedom given both in the arms and 
legs, the displacement of the point of interest on all axes, for the right upper extremity is given by

$$
\begin{gathered}
p_{x}=d c s X-a d_{2} \cos \left(\alpha_{u E r}\right) \cos \left(\beta_{u E r}\right)-a d_{3} \cos \left(\alpha_{I E r}\right) \cos \left(\beta_{I E r}\right) \\
p_{y}=a d_{2} \sin \left(\alpha_{u E r}\right) \cos \left(\beta_{u E r}\right)+a d_{3} \sin \left(\alpha_{I E r}\right) \cos \left(\beta_{I E r}\right) \\
p_{z}=d c s Z+a d_{2} \sin \left(\alpha_{u E r}\right)+a d_{3} \sin \left(\alpha_{I E r}\right),
\end{gathered}
$$

where $d c s X$ is the distance between the center of the thorax and the shoulder; $d c s Z$ is the distance between the abdomen and the center of the thorax; $a d_{2}$ is the distance between the shoulder and the elbow, $a d_{3}$ is the distance between the elbow and the point of interest (right hand); $\alpha_{u E r}$ it is a rotation angle of the shoulder, $\alpha_{I E r}$ is an angle of rotation of the elbow; $\alpha$ rotates on the $Z$ axis y $\beta$ rotates on the $X$ axis. The negative sign for the $X$ axis alternate the result of $\cos \left(\alpha_{u E \text { max }}\right)$, where $\alpha_{u E r \max }=\pi$.

For the left hand, the $\alpha_{u E l \max }=0$, therefore, the use of the negative sign is not necessary. The same criteria are used for the lower extremities.

In this way, the position of the point of interest can be determined in any of the axes of the space. The location of the operative end allows obtaining input data to the pattern comparator, which validates the correct execution of exercises.

\subsection{Robot extremities controller}

Considering the importance of the reference movements for the child to imitate them, a controller based on inverse kinematics is proposed to validate the movements of the extremities of the robotic mechanism. As shown in Fig.6, the degrees of freedom of the uppers and lowers extremities of the Bioloid suggest the number of $n$ equations and the number of $m$ unknowns for the design of the controller. In this way, a system of three equations and three unknowns presented in (4), (5), and (6) solve the problem of positioning the points of interest of the upper extremities at any point in space.

The inverse kinematics is achieved with control purposes for the robot, in addition to ensuring that the end of the robot executes desired movements identifying some type of error in the intelligent motors that execute the movement. 


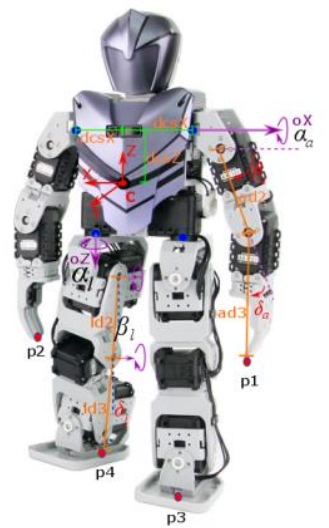

Fig.6. Degrees of freedom considered in the humanoid robot

$$
\begin{gathered}
p_{x}=d c s X+a d_{2} \cos \left(\beta_{a}\right)+a d_{3} \cos \left(\beta_{a}+\delta_{a}\right) \\
p_{y}=\cos \left(\alpha_{a}\right) \cdot\left(a d_{2} \sin \left(\beta_{a}\right)+a d_{3} \sin \left(\beta_{a}+\delta_{a}\right)\right) \\
p_{z}=h_{c}+d c s Z+\sin \left(\alpha_{a}\right)\left(a d_{2} \sin \left(\beta_{a}\right)+a d_{3} \sin \left(\beta_{a}+\delta_{a}\right)\right)
\end{gathered}
$$

Remark 2: To obtain the required models, it is considered that all extremities are formed with the same number of degrees of freedom and similar behavior, with the only difference that the base of each limb has a displacement from a given reference center. Likewise, it is considered that the initial position of the robot has the upper extremities completely stretched on the $X$ axis, while the lower extremities are stretched on the $Y$ axis.

Being based on mathematical methods and trigonometric properties, the angles of $\alpha$, $\beta$, and $\delta$ are shown in (7), (8), and (9).

$$
\begin{gathered}
\alpha_{a}=\tan ^{-1}\left(\frac{p_{z}-h_{c}-d c s Z}{p_{y}}\right) \\
\delta_{a}=\cos ^{-1}\left(\frac{\left(p_{z}-h_{c}-d c s Z\right)^{2}+p_{y}^{2}+\left(p_{x}-d_{x}\right)^{2}-a d_{2}^{2}-a d_{3}^{2}}{2 a d_{2} a d_{3}}\right) \\
\beta_{a}=\tan ^{-1}\left(\frac{p_{x}-d s c X}{\sqrt{\left(p_{z}-h_{c}-d s c Z\right)^{2}+p_{y}^{2}}}\right)-\tan ^{-1}\left(\frac{d_{3} \sin \left(\delta_{a}\right)}{d_{2}+d_{3} \cos \left(\delta_{a}\right)}\right)
\end{gathered}
$$

The mathematical representations found are based on the position of the extreme of interest $p_{x}, p_{y} \mathrm{y} p_{z}$, therefore, the displacement of a vector with positions will force the actuators to move the point of interest over the space, executing the desired therapy movements.

Bearing in mind that the vector of pattern movements is made up of a number of sequential points (movements in $X, Y$ and $Z$ desired), the objective of the controller is to modify the positions of the points of interest (hands and feet of the robot) depending on the new positions given by the reference vector. In this aspect, when a new desired point is proposed $\mathbf{p}_{\mathbf{d}}=\left[p_{x d}, p_{y d}, p_{z d}\right]$, the controller reaches the objective through 
the calculation of errors $\mathbf{p}_{\text {err }}=\mathbf{p}_{\mathbf{d}}-\mathbf{p}_{\mathbf{r}}$, where $\mathbf{p}_{\text {err }}=\left[p_{\text {err } X}, p_{\text {err } Y}, p_{\text {err }}\right]$, where, $\mathbf{p}_{\text {err }}$ represents the position error of the three components. The reduction of position errors is achieved with (10), where the use of a pair of constants is included $\mathbf{k}_{\mathbf{1}}$ and $\mathbf{k}_{2}$.

$$
\mathbf{p}_{i}=\mathbf{p}_{i-1}+\mathbf{p}_{\text {err }} *\left(\mathbf{k}_{\mathbf{1}}+\mathbf{k}_{\mathbf{2}}\right)
$$

The response of the controller to achieve the desired new position can force the actuators exceedingly. To adjust the responses of the controller to rotation values that do not affect the structure, the use of the constants is proposed $\mathbf{k}_{\mathbf{1}}$ and $\mathbf{k}_{\mathbf{2}}$. For normal execution, $\mathbf{k}_{\mathbf{1}}$ is included to provide the system with a basic speed, while through the use of $\mathbf{k}_{2}$, the user can modify the speed of execution to its need.

Remark 3: The implementation of the system includes four controllers, one for each extremity running simultaneously, which accelerates the error correction process given a vector of desired positions. For more information about the robotic platform used visit the following page http://www.robotis.us/bioloid-1/

\subsection{Comparison of patterns through DTWv}

The parallelism of the FPGA where the system is implemented facilitates the incorporation of four vector alignment and comparison algorithms. The cited vectors contain spatial positions $X, Y, Z$ of the four points of interest (two hands, two feet), identifying as the pattern vector the number of desired spatial points, while the input vector contains the positions of the points of interest, obtained from the patient (by using inertial measurement sensors and processing by direct kinematics). For the development of the DTW in the FPGA, fundamental aspects are considered [7]: boundary condition, condition of monotony, adjustment window condition, and jump condition. The boundary condition states that the trajectory starts at the bottom right and ends at the top left; the condition of monotony, refers to the progress in the search for solutions, where in no case the trajectory can go backwards, only maintain or increase the values of index $i$ and $j$; the jump condition, forces the trajectory to advance one step at a time; and finally, the adjustment window condition, establishes that the trajectory in shown Fig. 8. it should be not too far from the diagonal.

The programming of the algorithm yields a matrix of $2 \times n$ values, where the path that determines the similarity between both frames is defined. The results section includes the usability of the programmed algorithm. The determination of the path additionally allows to know a total of the weight matrix, whereby decision thresholds are defined to compare the input patterns. 


\section{$4 \quad$ Results}

The results of the proposal are split into three parts, starting with the test of data acquisition on the patient's movements through direct kinematics and the use of inertial sensors. On the other hand, the second group of results presents the correct execution of reference movements by the robotic mechanism, validating the proposed controller. Finally, the alignment of the movement vectors is presented to determine the coincidences between the movements executed by the robot (reference movements) and the movements of the child.

The $i$ ) child's movement information acquisition tests are shown in this subsection. Fig.7 presents the recreation of positions of each of the extremities of the child in Python, processing which takes place on a Raspberry processor. In this way and using the methods previously indicated, the movements of the points of interest (both hands and feet) are determined.

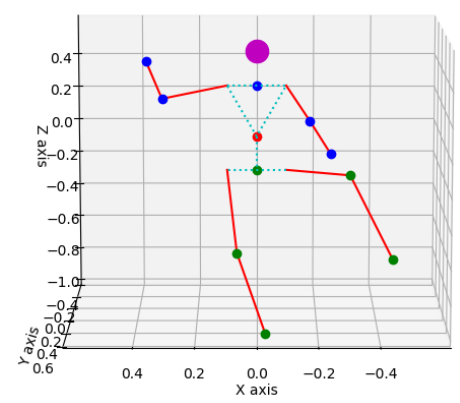

Fig. 7. Representation of the positioning of the extremities in the auxiliary processor

On the other hand, ii) the correct execution of movements by the robotic mechanism is crucial to motivate the child to execute the exercises in the expected manner. The validation of the algorithm which controls the proposed limb is based on the results shown in Fig. 8, where the correction of positions through the convergence of errors to zero in each of the desired positions is observed.

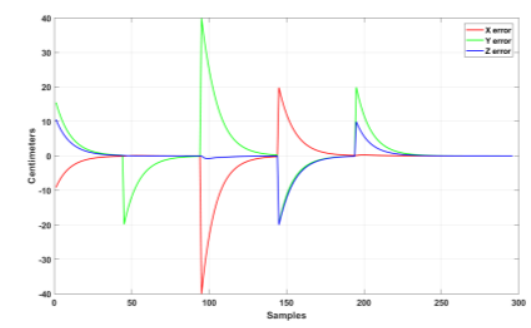

Fig. 8. Correction of errors in each of the axes 
Likewise, the iii) alignment between the reference vectors and the input vectors is presented in this section through the distance matrix, the total weight, and the aligned vectors. Considering that the comparison is between two experiments in which the satisfactory and wrong movements of the user's right hand are compared (Fig. 9), the alignment results are shown in this subsection.

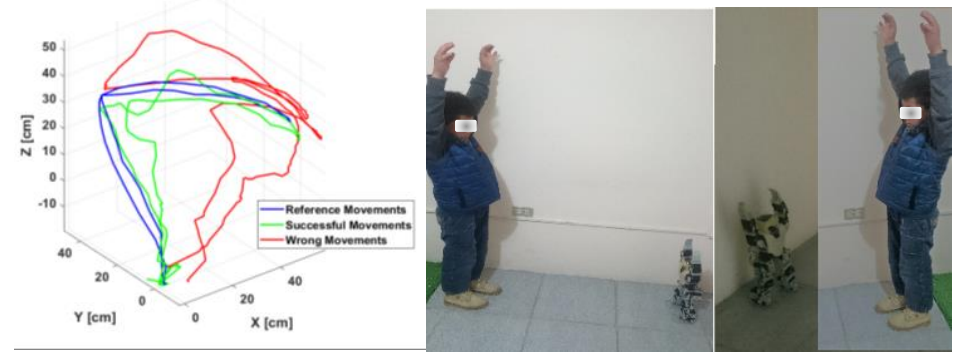

Fig. 9. Movements of the user's right hand

The representation of the distance matrix presented in Fig. 10 facilitate the interpretation of the results obtained, where the trend of dark colors in the diagonal of the first image indicates that the compared vectors have a certain similarity despite the fact that the amount of data is not similar. On the other hand, the second image shows a low tendency of negative colors on the diagonal, which indicates at first glance the nonsimilarity between the input vectors. This information is of great importance for the feedback of the system, since depending on the distance matrix, the total distance used to send commands to the auxiliary processor that interprets this information and generates audio outputs to motivate the child is obtained and comply with the exercises proposed in the therapy.

Remark 4: Based on experimentation of this work, the reference vectors of the three components form a single vector (in the order $x, y, z$ ), in the same way as the vectors read. In this way, a pair of vectors are formed and compared to obtain the result of the DTW as shown in Fig.10, Fig. 11 and Fig.12.

Remark 5: The topic is open for future research considering that the system has been tested with healthy children.

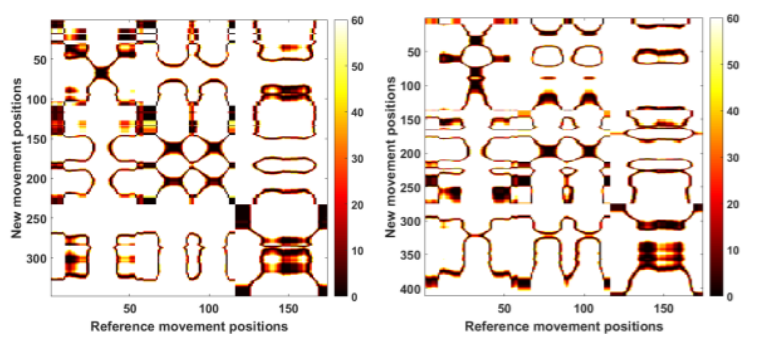

Fig. 10. Distance matrix 


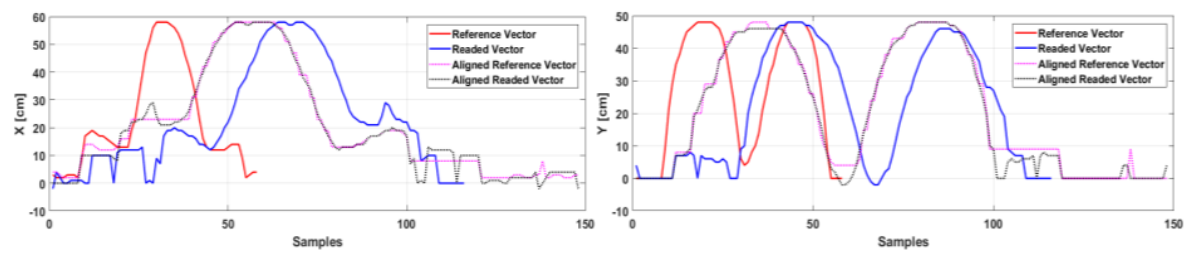

Fig.11. DTW response to similar sequence
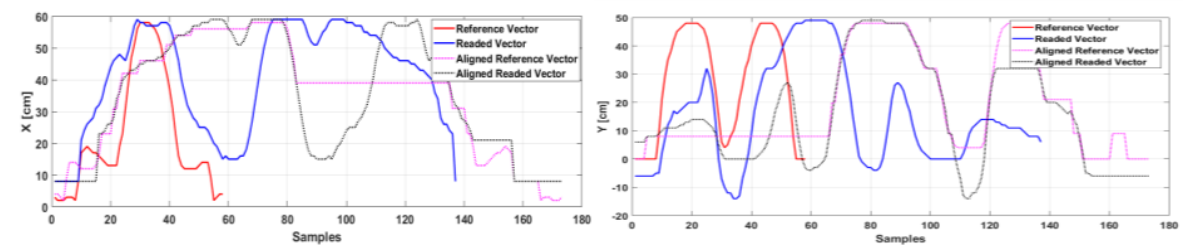

Fig. 12. DTW responce to different sequence

\section{Conclusions}

This work presents a strategy of non-invasive rehabilitation for children, inte-grating a humanoid robotic mechanism, inertial sensors, and comparative and vector alignment techniques. The system is supervised by the therapist, being a support tool in rehabilitation activities. The main purpose of this system is to provide the child with a more interactive environment through the use of the humanoid, where the user must replicate the movements of the robot to obtain a satisfactory result. To determine if the task is performed in the expected manner, the FPGA compares the pattern movements with movements generated by the patient through a temporal alignment algorithm (DTW), returning a sound response to motivate a new execution or validate the task done. Additionally, experimental results to validate the correct execution of movements of the limbs of the robot are presented, demonstrating the tendency of errors to zero in the positioning of the points of interest.

As future works, it is proposed to modify the structure of the humanoid in order to incorporate a camera in the head of the robotics mechanism and through artificial vision to implement the interactive rehabilitation system.

\section{References}

1. World Health Organization: WHO. (2011). WORLD REPORT DISABILITY. Landed https://www.who.int/disabilities/world_report/2011/summary_es.pdf?ua=1

2. INEC. (s.f.). Health Activities and Resources» Creative Commons, 2015. Landed http://www.ecuadorencifras.gob.ec/actividades-y-recursos-de-salud-2015/

3. CONADIS. (2018). National Council for the Equality of Disabilities. Landed https://www.consejodiscapacidades.gob.ec/estadisticas-de-discapacidad 
4. Milligan, L. (8 de 7 de 2017). Advancements in PT Tech/Emerging Trends. Landed: WebPT: http://www.webpt.com/blog/post/advancements-pt-techemerging-trends

5. C. Krista, L. Corinna y K. Kenton, «Development of an Interactive Upper Extremity Gestural Robotic,» Annual International Conference of the IEEE EMBS, pp. 5973-5976, (2009).

6. P. Pawel, W. David, C. Edith, H. Yves, H. Lisa y F. Ismael, «A Paediatric Interactive Therapy System for Arm and Hand,» 2008 Virtual Rehabilitation, pp. 127-132, (2008).

7. S. Piedad A, A. Víctor H, L. Johana, C. Fernando A. y A. Andrés, «Visual feedback framework for rehabilitation of stroke patients,» Informatics in Medicine Unlocked, vol. 13, pp. 41-50, (2018).

8. J. Acevedo, E. Caicedo y J. Castillo, «Application of robotics rehabilitation technologies,» Revista Universidad Industrial de Santander Salud, vol. 49, n 1, pp. 103-114, (2017).

9. A. Billard, B. Robins, J. Nadel y K. Dautenhahn, «Building Robota, a Mini-Humanoid Robot for the Rehabilitation of Children With Autism,» Assistive Technology, vol. 19, $\mathrm{n}^{\circ} 1$, pp. 37-49, (2010).

10. B. Robins, K. Dautenhahn, R. Boekhorst y A. Billard, «Robotic assistants in therapy and education of children with autism: can a small humanoid robot help encourage social interaction skills?,» Universal Access in the Information Society, vol. 4, pp. 105-120, (2005).

11. S. Syamimi, Y. Hanafiah, I. Luthffi, H. Fazah Akhtar, M. Salina y A. P. Hanizah, «Initial Response of Autistic Children in Human-Robot Interaction Therapy with Humanoid Robot NAO,» de IEEE 8th International Colloquium on Signal Processing and its Applications, Malaysia, (2012).

12. J. E. Maja J Matarić, D. J. Feil-Seifer y C. J. Winstein, «Socially assistive robotics for poststroke rehabilitation,» Journal of NeuroEngineering and Rehabilitation, vol. 4, $\mathrm{n}^{\circ}$ 5, pp. 29, (2007).

13. L. Calderita Estévez, P. Bustos García, C. Suárez Mejías, F. Fernández Rebollo y A. Bandera Rubio, «THERAPIST: Towards an Autonomous Socially Interactive Robot for Motor and Neurorehabilitation Therapies for Children,» 2013 7th International Conference on Pervasive Computing Technologies for Healthcare and Workshops, pp. 374-377, (2013). 